MZ-TH/98-59

December 1998

\title{
On the running electromagnetic coupling constant at $M_{Z}$
}

\author{
J.G. Körner ${ }^{1}$, A.A. Pivovarov ${ }^{1,2}$ and K.Schilcher ${ }^{1}$ \\ ${ }^{1}$ Institut für Physik, Johannes-Gutenberg-Universität, \\ Staudinger Weg 7, D-55099 Mainz, Germany \\ ${ }^{2}$ Institute for Nuclear Research of the \\ Russian Academy of Sciences, Moscow 117312
}

\begin{abstract}
We present a discussion on how to define the running electromagnetic coupling constant at $M_{Z}$ or some other intermediate scale as e.g. $m \Upsilon$. We argue that a natural definition consistent with general requirements of the renormalization group should be based on Euclidean values of the momentum of the photon propagator as the appropriate scale. We demonstrate in an explicit example of evaluating the running coupling constant at the scale of the $\Upsilon$ resonance mass that the usual definition of the hadronic contribution with a principal value prescription is inconsistent. In the determination of the value of $\alpha$ at $M_{Z}$ the numerical difference due to using a Euclidean definition rather than the principal value one is comparable in size to the errors caused by existing experimental and QCD inputs to the evaluation of $\alpha\left(M_{Z}\right)$.
\end{abstract}


In applications to high precision tests of the standard model [四] with observables near the $Z$ boson peak the electromagnetic coupling constant should be used at a scale of the order of the $Z$ boson mass $M_{Z}$, (see e.g. [2, 3]). The running electromagnetic coupling constant at $M_{Z}$ has even been chosen as a standard reference parameter [4]. It differs numerically from the value of the fine structure constant $\alpha^{-1}=137.036 \ldots$ defined at zero momentum or from the Coulomb law for heavy nonrelativistic particles. The change is usually accounted for through the renormalization group equation [5, 6]. Because the fine structure constant is defined at vanishing momentum and is taken as initial value in the solution of the renormalization group equation, the running electromagnetic coupling constant at $M_{Z}$ is an infrared sensitive quantity in as much as the contribution of strong interaction is not easy to compute due to the nonperturbative region at small energies. Therefore this contribution is usually taken into account in the leading order of electromagnetic interaction within a semiphenomenological approximation through a dispersion relation. There has been a renewal of interest in a precise determination of the hadronic contribution during the last years in particular in connection with the constraints on the Higgs boson mass [7]. Some recent references giving a state-of-the-art analysis of this contribution are [8, 9, 10, 11]. A quasi-analytical approach was used in [12] where some references to earlier paper can be found (see also [13, 14]). An extremely thorough data-based analysis is given in 15 .

In the present note we critically discuss the definition of the running electromagnetic coupling constant at $M_{Z}$ as it is used in the literature. The standard approach consists in using the principal value prescription at the appropriate scale in the physical domain on the positive energy semiaxis. We argue that a natural definition consistent with general requirements and the standard notion of running used in renormalization group applications should be based on the Euclidean momentum of 
the photon propagator as the appropriate scale.

The running coupling $\alpha\left(q^{2}\right)$ is defined through the (one-photon irreducible) photon vacuum polarization function $\Pi_{\gamma}\left(q^{2}\right)$ as

$$
\alpha\left(q^{2}\right)=\frac{\alpha}{1-\Pi_{\gamma}\left(q^{2}\right)} .
$$

$\Pi_{\gamma}\left(q^{2}\right)$ contains both leptonic and hadronic contributions. The hadronic part of the polarization function $\Pi_{\gamma}\left(q^{2}\right)$ (with one subtraction at zero momentum) reads

$$
\Pi_{\gamma}^{h a d}\left(q^{2}\right)=-\frac{\alpha}{3 \pi} q^{2} \int_{4 m_{\pi}^{2}}^{\infty} \frac{R_{h}(s) d s}{s\left(s-q^{2}-i 0\right)}
$$

where $R_{h}(s)$ is the normalized cross section of $e^{+} e^{-}$annihilation into hadrons. Let us introduce, for convenience, the polarization function $\Pi\left(q^{2}\right)$

$$
\Pi\left(q^{2}\right)=-q^{2} \int_{0}^{\infty} \frac{R(s) d s}{s\left(s-q^{2}-i 0\right)}
$$

such that

$$
\Pi_{\gamma}\left(q^{2}\right)=\frac{\alpha}{3 \pi} \Pi\left(q^{2}\right)
$$

where $R(s)$ is the corresponding spectral density. Note that $\alpha\left(q^{2}\right)$ is defined for every complex value of $q^{2}$ by eq. (2) . For real negative $q^{2}$ the polarization function $\Pi_{\gamma}\left(q^{2}\right)$ (and $\Pi\left(q^{2}\right)$ as well) is a positive real number because the spectral density $R(s)$ is positive.

The definition (何) is used in renormalization group applications and the scale $q^{2}$ is taken to be a real negative number which corresponds to a propagator in the Euclidean domain. The Euclidean definition is mainly used in applications of grand unified theories [16], supersymmetry at large energy [17], physics at the Planck's scale etc.

For the precise study of the physics at the $Z$ boson pole the effective electromagnetic interaction coupling constant $\bar{\alpha}$ is represented in the form

$$
\bar{\alpha}=\frac{\alpha}{1-\Delta \alpha}
$$


Numerically one obtains a real positive number for $\Delta \alpha$.

A theoretical expression for $\Delta \alpha$ is defined in the present literature directly on the positive semiaxis by making use of the principal value prescription for the singularity of the integrand in eq. (2)

$$
\Delta \alpha=\operatorname{Re} \Pi_{\gamma}\left(M_{Z}^{2}\right), \quad \operatorname{Re} \Pi_{\gamma}\left(M_{Z}^{2}\right)=-\frac{\alpha}{3 \pi} M_{Z}^{2} \mathrm{P} \int_{4 m_{\pi}^{2}}^{\infty} \frac{R(s) d s}{s\left(s-M_{Z}^{2}\right)} .
$$

Here $\mathrm{P} \int$ denotes the principal value of the integral. This makes $\Delta \alpha$ real (the initial $i 0$ prescription for the integral gives it an imaginary part) which is appropriate for a coupling constant. We argue that this prescription is not adequate for the physical situation at hand and does not correspond to the notion of a running coupling used in the standard renormalization group applications. The latter corresponds to scales taken in the Euclidean domain

$$
\alpha_{E}\left(\mu^{2}\right)=\frac{\alpha}{1-\Pi_{\gamma}\left(-\mu^{2}\right)}, \quad \Pi_{\gamma}\left(-\mu^{2}\right)=\frac{\alpha}{3 \pi} \mu^{2} \int_{4 m_{\pi}^{2}}^{\infty} \frac{R(s) d s}{s\left(s+\mu^{2}\right)} .
$$

The running electromagnetic coupling constant at the scale $M_{Z}$ is defined then as $\alpha_{E}\left(M_{Z}^{2}\right)$. Therefore, for the phenomenological parameter "running electromagnetic coupling constant at the scale $M_{Z}$ " denoted by $\bar{\alpha}$ we have two representations:

i) the standard one with a principal value prescription

$$
\bar{\alpha}=\alpha_{P V}\left(M_{Z}^{2}\right), \quad \Delta \alpha=\operatorname{Re} \Pi_{\gamma}\left(M_{Z}^{2}\right)
$$

ii) the alternative one in the Euclidean domain

$$
\bar{\alpha}=\alpha_{E}\left(M_{Z}^{2}\right), \quad \Delta \alpha=\Pi_{\gamma}\left(-M_{Z}^{2}\right)
$$

which is defined through

$$
\alpha_{E}\left(M_{Z}^{2}\right)=\frac{\alpha}{1-\Pi_{\gamma}\left(-M_{Z}^{2}\right)}, \quad \Pi_{\gamma}\left(-M_{Z}^{2}\right)=\frac{\alpha}{3 \pi} M_{Z}^{2} \int_{4 m_{\pi}^{2}}^{\infty} \frac{R(s) d s}{s\left(s+M_{Z}^{2}\right)} .
$$


We suggest that the Euclidean version is used. The idea of changing scales is embodied in the renormalization group equation which allows one to control large logarithms. Therefore theoretically one is dealing with a logarithm of the ratio of two scales. Note that the notion of scale becomes rather imprecise as soon as complex numbers are involved. For example, numerically $M_{Z}^{2}$ has the same 'scale' as $e^{i \pi} M_{Z}^{2}=$ $-M_{Z}^{2}$. The choice of an appropriate scale is determined by the particular kinematics and the higher order corrections of each particular process in question. In the leading logarithmic approximation, however, keeping the finite corrections to large logarithms is beyond the accuracy of the approximation that renders all scales with the same absolute value equivalent. As a reference value for the coupling constant the usual choice is to take a Euclidean point. This problem was discussed for strong interactions in [18] where different versions of a real part or absolute value definition of the coupling constant at complex points have been also studied. In the general case it is difficult to decide on how to deal with observables including complex numbers within the renormalization group resummation of logarithms. For two-point functions, however, there is a natural solution to this problem based on their analytic properties given by the dispersion representation [19, 20].

Below we discuss these two possibilities of defining the "running electromagnetic coupling constant at the scale $M_{Z}$ ".

First we show that the two definitions (Euclidean and principal value) are close numerically for applications in the vicinity of the $Z$ boson peak discussed in the literature. Let us take eq. (2) and split the whole region of integration into two parts separated by $s_{0}$

$$
\Pi\left(q^{2}\right)=-q^{2} \int_{0}^{s_{0}} \frac{R(s) d s}{s\left(s-q^{2}-i 0\right)}-q^{2} \int_{s_{0}}^{\infty} \frac{R(s) d s}{s\left(s-q^{2}-i 0\right)} .
$$

If $\left|q^{2}\right|$ is chosen such that $\left|q^{2}\right| \gg s_{0}$ one can expand the denominator in the first integral. Then, if $s_{0}$ is large enough one can use perturbation theory for the spectral 
density in the second integral. For illustrative purposes we choose a very simplified approximation for $R(s)$, namely $R(s)=$ const $=1$ for $s>s_{0}$. Then one obtains

$$
\Pi\left(q^{2}\right)=\int_{0}^{s_{0}} \frac{R(s)}{s} d s+\ln \frac{\left|s_{0}-q^{2}\right|}{s_{0}}
$$

where the principal value prescription has been used. Expanding eq. (12) in the limit $\left|q^{2}\right| \gg s_{0}$ one finally obtains

$$
\Pi\left(q^{2}\right)=\int_{0}^{s_{0}} \frac{R(s)}{s} d s+\ln \frac{\left|q^{2}\right|}{s_{0}}
$$

which is independent of the phase of the complex number $q^{2}$. The same result can be obtained also directly from eq. (3) in this limit. Therefore in the above approximation with the suggested regime of variables both the Euclidean and principal value definitions are equivalent numerically. Later on we discuss corrections to this leading order approximation which depend on whether the Euclidean and principal value definition is used.

This is a qualitative picture. Because the above simplifying assumptions can be expected to correctly embody the main features of a more sophisticated numerical analysis it is clear that the numerical change stemming from the choice of $q^{2}$ in the Euclidean domain rather than using the conventional definition is under control at the scale of $M_{Z}$ and does not jeopardize current phenomenology. However, the definition in the Euclidean domain given in eq. (10) is preferable from a theoretical point of view. It is natural. It gives a real number. It is smooth. It is consistent with the renormalization group.

And the principal value prescription has equally obvious deficiencies. It is ad hoc. It gives a real part of a propagator which is not directly related to a coupling constant in a renormalization group sense. It is not smooth.

The last deficiency is, in fact, the most crucial one. Let us present more details. We take the principal value definition at $q^{2}=M_{Z}^{2}$ and compute the polarization 
function for a model spectral density $R(s)=\theta\left(s-s_{0}\right)$ obtaining

$$
\Pi\left(M_{Z}^{2}\right)=\ln \frac{\left|s_{0}-M_{Z}^{2}\right|}{s_{0}} .
$$

Note first that the polarization function (14) gives a rather curious result

$$
P \int_{M_{Z}^{2} / 2}^{\infty} \frac{d s}{s\left(s-M_{Z}^{2}\right)}=0
$$

which means that the contribution of all states with masses larger than $M_{Z} / \sqrt{2} \sim$ $60 \mathrm{GeV}$ is exactly equal to zero assuming the asymptotic spectral density in this region to be a constant. Also there is a sign change in the vicinity of $M_{Z} / \sqrt{2}$. This feature persists for any realistic $R(s)$ in the vicinity of some point $s^{*} \simeq M_{Z}^{2} / 2$ because in this region QCD perturbation theory works well and the spectral density is rather smooth and close to its asymptotic value which is almost a constant (up to a slow logarithmic decrease). Therefore one gets the exact equality

$$
P \int_{s^{*}}^{\infty} \frac{R(s) d s}{s\left(s-M_{Z}^{2}\right)}=0
$$

for some $s^{*} \sim M_{Z}^{2} / 2$.

Furthermore, if one takes $s_{0}=M_{Z}^{2}$ in eq. (14) then the logarithm is ill-defined. This can be seen to be a consequence of the principal value definition eq. (6). Even if this is a rather academic example we nevertheless take it as a warning (because there is no sharp increase of the spectral density or changes in general in the vicinity of the $Z$ boson mass). More realistic situations are considered below. In contrast to the principal value definition the Euclidean definition is fine also in this case

$$
\Pi\left(-M_{Z}^{2}\right)=\ln \frac{s_{0}+M_{Z}^{2}}{s_{0}} .
$$

The reason for the ill-defined behavior of eqs. (14) and (6) is clear. The principal value prescription leads to a distribution $P \frac{1}{x}$ which is defined only on smooth functions. A 
product of two distributions

$$
\left(P \frac{1}{s-M_{Z}^{2}}\right) \theta\left(s-M_{Z}^{2}\right)
$$

is not an integrable function. An ad hoc definition with a principal value prescription fails to define a value for the running coupling at some particular points and one has to introduce further rules for such cases.

Also in a more realistic situation one needs the running electromagnetic coupling constant at the scales around the masses of resonances of the $J / \psi$ or $\Upsilon$ families to account for their leptonic widths. With the principal value definition it is impossible to compute the running electromagnetic coupling constant at the scales around the resonance masses. Indeed, the correction $\Delta \alpha$ to the running electromagnetic coupling constant at the scale of the $\Upsilon$ meson mass $m_{\Upsilon}$ is given by an ill-defined integral of the product of two distributions

$$
\left(P \frac{1}{s-m_{\Upsilon}^{2}}\right) \delta\left(s-m_{\Upsilon}^{2}\right)
$$

This quantity is not defined as a distribution for the same reason as a product of two distributions is not defined when their singular points coincide.

Leaving the mathematical statement about the ill-defined behavior of a product of two distributions aside, in practice (for finite widths of the resonances or for some sharp but still smooth increase of the spectral function) the results following from the principal value definition will be unstable. As an explicit example we take the spectral density corresponding a single Breit-Wigner resonance and calculate its contribution to $\operatorname{Re} \Pi\left(M_{Z}^{2}\right)$. The Breit-Wigner spectral function is given by

$$
R_{B W}(s)=\frac{1}{\pi} \frac{\Gamma M}{\left(s-M^{2}\right)^{2}+\Gamma^{2} M^{2}}
$$

where $M$ and $\Gamma$ are the mass and the width of the resonance. As $\Gamma \rightarrow 0$ one obtains $R_{B W}(s) \rightarrow \delta\left(s-M^{2}\right)$. After integration (with proper care for the point $s=0$ ) one 
finds

$$
M_{Z}^{2} \mathrm{P} \int^{\infty} \frac{R_{B W}(s) d s}{s\left(s-M_{Z}^{2}\right)}=\frac{M^{2}-M_{Z}^{2}}{\left(M^{2}-M_{Z}^{2}\right)^{2}+\Gamma^{2} M^{2}}+\ldots=\frac{\Delta}{\Delta^{2}+\Gamma^{2} M^{2}}+\ldots
$$

with $\Delta=M^{2}-M_{Z}^{2}$. Only potentially singular terms for the limit $\Gamma \rightarrow 0$ in the vicinity $M^{2} \sim M_{Z}^{2}$ have been kept. The last function in eq. (21) has its extremal points at $\Delta= \pm \Gamma M$ with the values

$$
\left.\frac{\Delta}{\Delta^{2}+\Gamma^{2} M^{2}}\right|_{\Delta= \pm \Gamma M}= \pm \frac{1}{2 \Gamma M} .
$$

There is no regular limit $\Gamma \rightarrow 0$ and further rules would be required to deal with this limit. Note that the result (21) can be obtained without explicit integration of the Breit-Wigner spectrum. Since the Breit-Wigner function can be regarded as a regularization of a $\delta$-distribution one can introduce a regularization of $P \frac{1}{x}$ in the class of infinitely smooth function instead. An example is

$$
\lim _{\epsilon \rightarrow 0} \frac{x}{x^{2}+\epsilon^{2}}=P \frac{1}{x} \quad \text { and } \quad \lim _{\epsilon \rightarrow 0} \frac{\left(s-M_{Z}^{2}\right)}{\left(s-M_{Z}^{2}\right)^{2}+\epsilon^{2}}=P \frac{1}{s-M_{Z}^{2}} .
$$

Then after the integration with an infinitely narrow resonance one gets

$$
M_{Z}^{2} \int \frac{\delta\left(s-M^{2}\right)\left(s-M_{Z}^{2}\right) d s}{s\left[\left(s-M_{Z}^{2}\right)^{2}+\epsilon^{2}\right]}=\frac{M_{Z}^{2}}{M^{2}} \frac{\Delta}{\Delta^{2}+\epsilon^{2}}=\frac{\Delta}{\Delta^{2}+\epsilon^{2}}+\ldots
$$

for $M^{2} \sim M_{Z}^{2}$. The regularization can not be unambiguously removed, i.e. there is no unique limit at $\epsilon=0$ in the vicinity of $\Delta=0$. Of course, this is a reflection of the fact that the product of two distributions is ill-defined.

We will not dwell on the ill-defined behavior when a $\theta$-function type spectral density is used. This is a realistic situation when one computes the light quark contributions to the running electromagnetic coupling constant normalized in the vicinity of a sharp raise of the spectral density around $1.5 \mathrm{GeV}^{2}$.

With a Euclidean definition none of the above difficulties appear. Also because in this case the polarization function is defined in the Euclidean domain one need not 
integrate all data (only a small region near the origin requires explicit integration) and can use all the power of perturbation theory (e.g. [21). Although special care has to be taken about the subtraction at zero momentum that enters the definition of the coupling constant and makes it an infrared sensitive quantity. For this purpose, however, more sophisticated means can be used that will increase the accuracy [22, 23, 24].

We add two further remarks. The first concerns the leptonic contribution. For a lepton with the mass $m_{l}$ the asymptotic form of this contribution reads

$$
\Pi_{l e p t}=\ln \left(\frac{M_{Z}^{2}}{m_{l}^{2}}\right)-\frac{5}{3}
$$

and has the same real part for any real phase $\varphi$ of $e^{i \varphi} M_{Z}^{2}$. When the asymptotic form is used both prescriptions are equivalent numerically.

The second remark is related to the higher order contributions of the electroweak interactions. In the next order of the electroweak interaction there is a contribution of the $Z$ boson peak to the polarization function $\Pi_{\gamma}\left(q^{2}\right)$ due to $\gamma Z$ transitions (e.g. [25]). Therefore in that order one has to interpret the product of the principal value distribution with the sharp Breit-Wigner spectrum of the $Z$ boson pole itself. Again this problem is not present within the Euclidean definition.

Now we discuss very briefly the numerical difference that can result from the change of the definition of the running coupling constant at $M_{Z}$. Our model for the hadronic spectral density $R(s)$ is simple and is mainly designed for illustrative purposes such that one can easily trace the difference between the principal value and Euclidean definitions of the running coupling constant. The spectral density is chosen such that all calculations can be done analytically which is convenient for the purpose of estimating the order of magnitude of the difference in the two definitions. For the light quarks $u, d, s$ we assume the existence of a low lying resonance (like $\rho$, $\omega, \varphi)$ and a continuum. In general, we take the following form of the spectrum for 
every light quark flavor

$$
R_{\text {light }}(s)=3 Q_{q}^{2}\left[2 m^{2} \delta\left(s-m^{2}\right)+\theta\left(s-2 m^{2}\right)\right]
$$

according to the model of ref. [26] with $Q_{q}$ being a light quark fractional charge. The coupling of the low lying resonances have been replaced by the duality interval $2 \mathrm{~m}^{2}$. For heavy quarks we take the simplest model of the form

$$
R_{\text {heavy }}(s)=3 Q_{Q}^{2} \theta\left(s-4 m_{Q}^{2}\right)
$$

which represents the partonic asymptotic value with a naive step function two-quarkthreshold with $Q_{Q}$ being a heavy quark charge. Collecting everything together we find the following results. The three light quarks give the result for $\Pi\left(q^{2}\right)$ in the general form

$$
\Pi_{\text {light }}\left(q^{2}\right)=2\left(\frac{-2 q^{2}}{m^{2}-q^{2}-i 0}+\ln \frac{\left|2 m^{2}-q^{2}\right|}{2 m^{2}}\right) .
$$

Expanding at $\left|q^{2}\right| \sim M_{Z}^{2}$ one gets

$$
\Pi_{\text {light }}=2\left[2+\ln \frac{\left|M_{Z}^{2}\right|}{2 m^{2}}+O\left(m^{6} / M_{Z}^{6}\right)\right]
$$

which gives the same answer for both definition with a high precision. The difference starts at the order $O\left(\mathrm{~m}^{6} / M_{Z}^{6}\right)$ and is completely negligible for light resonance masses of order $1 \mathrm{GeV}(\rho-, \omega-, \varphi$-resonances, for instance). For the charm contribution we find

$$
\Pi_{\text {charm }}^{P V}=\frac{4}{3} \ln \frac{M_{Z}^{2}-4 m_{c}^{2}}{4 m_{c}^{2}}
$$

in the case of the principal value prescription. In the case of the Euclidean prescription one has

$$
\Pi_{\text {charm }}^{E}=\frac{4}{3} \ln \frac{M_{Z}^{2}+4 m_{c}^{2}}{4 m_{c}^{2}} .
$$

Expanding these formulae in the small ratio $4 m_{c}^{2} / M_{Z}^{2}$ one finds in the leading order

$$
\Pi_{\text {charm }}^{P V, E}=\frac{4}{3} \ln \frac{M_{Z}^{2}}{4 m_{c}^{2}}\left(1 \mp \frac{4 m_{c}^{2}}{M_{Z}^{2}} \frac{1}{\ln \frac{M_{Z}^{2}}{4 m_{c}^{2}}}\right) .
$$


We keep this form for further numerical comparison in the case of $b$ and $t$ quarks. For the $b$ quark contribution one gets

$$
\Pi_{\text {bottom }}^{P V, E}=\frac{1}{3} \ln \frac{M_{Z}^{2}}{4 m_{b}^{2}}\left(1 \mp \frac{4 m_{b}^{2}}{M_{Z}^{2}} \frac{1}{\ln \frac{M_{Z}^{2}}{4 m_{b}^{2}}}\right),
$$

and for the $t$ quark

$$
\Pi_{t o p}^{P V, E}=\frac{4}{3} \ln \left(1 \mp \frac{M_{Z}^{2}}{4 m_{t}^{2}}\right) .
$$

For numerical estimates we take $\sqrt{2} m=1 \mathrm{GeV}$ for the light quark resonances, $m_{c}=$ $1.4 \mathrm{GeV}, m_{b}=4.8 \mathrm{GeV}, m_{t}=175 \mathrm{GeV}, M_{Z}=91 \mathrm{GeV}$. Note that the exact definition of the quark mass parameters is not required here because it is far beyond the accuracy of our simple model. Even more, these parameters can be considered as effective parameters serving to describe integrals over the threshold regions of quark production. Nevertheless we stick to almost canonical values for the pole quark masses. While the absolute value of the contribution will be obtained rather approximately, the model however is fairly sufficient for our main purpose to estimate the difference between the two definitions. Numerically, eq. (25) leads to the light quark contribution

$$
\text { light quarks }(\mathrm{u}, \mathrm{d}, \mathrm{s})=2 \times(2+2 \times 4.5)=22.0
$$

with both prescriptions. For the contributions of the heavy quarks we find

$$
\begin{aligned}
\mathrm{c}-\text { quark } & =\frac{4}{3} \times 7.0\left(1 . \mp 0.14 \times 10^{-3}\right) \\
\mathrm{b}-\text { quark } & =\frac{1}{3} \times 4.5\left(1 . \mp 2.5 \times 10^{-3}\right) \\
\mathrm{t}-\text { quark } & =\frac{4}{3} \times\left(\mp 68 \times 10^{-3}\right) .
\end{aligned}
$$

Summing everything together one obtains

$$
22.0+\frac{4}{3} \times 7.0\left(1 . \mp 0.14 \times 10^{-3}\right)_{c}+\frac{1}{3} \times 4.5\left(1 . \mp 2.5 \times 10^{-3}\right)_{b}+\frac{4}{3} \times\left(\mp 68 \times 10^{-3}\right)_{t}
$$




$$
=32.8+0.1 \delta \approx 33+0.1 \delta
$$

where $\delta=-1$ for the principal value definition and $\delta=1$ for the Euclidean definition. One sees that the difference is saturated by the top quark contribution. This is natural because it has a mass closest to $M_{Z}$. Its contribution is small in absolute value but is completely different for the two definitions.

The lepton contribution is taken into account according to the asymptotic formula eq. (24) with $m_{e}=0.5 \mathrm{MeV}, m_{\mu}=0.1 \mathrm{GeV}, m_{\tau}=1.8 \mathrm{GeV}$ which gives

$$
(24.2)_{e}+(13.6)_{\mu}+(7.8)_{\tau}-5=40.6 \approx 41
$$

The final result for the total contribution of the charged fermions to the polarization function reads

$$
33+0.1 \delta+41=74+0.1 \delta
$$

Dividing by $3 \pi$ one obtains the total contribution to the inverse coupling constant

$$
(74+0.1 \delta) / 3 \pi=7.9+0.01 \delta
$$

For the inverse running electromagnetic coupling constant we obtain

$$
137.0-(7.9+0.01 \delta)=129.1-0.01 \delta
$$

Even though the central value of our approximate evaluation is rather close to the results of more precise evaluations [8, 9, 10, 11] this agreement should not be taken too seriously. Our estimate is rather rough and serves the purpose well to obtain the numerical change between the two definitions of the running electromagnetic coupling constant at $M_{Z}$. At present the change due to the different definitions has no influence on current phenomenology. This change is within the error bars for the uncertainty of the more precise values $128.93 \pm 0.06$ [9] or even with smaller errors $128.93 \pm 0.015_{\exp } \pm 0.015_{\text {th }}$ [8, 10, 11]. Therefore the Euclidean definition, which 
we consider more consistent theoretically, does not violate current phenomenology. However, the change is comparable in size with the present uncertainties and when the experimental data used in the determination of the running electromagnetic coupling constant at $M_{Z}$ improves in the future the difference between the two definitions will become significant.

Our last numerical example concerns the order of magnitude of the singular term in the coupling normalized at the scale $m_{\Upsilon}$ within the principal value prescription. With the same normalization as in our simple model the contribution of the $\Upsilon$-resonance to the spectral density in the Breit-Wigner approximation reads

$$
R_{\Upsilon}(s)=\frac{2}{3} m_{\Upsilon} \Delta_{\Upsilon} R_{B W}\left(s, m_{\Upsilon}, \Gamma\right)
$$

where $\Delta_{\Upsilon} \approx 1 \mathrm{GeV}$ is its duality interval in energy units [27] related to its leptonic decay width, $\Delta_{\Upsilon}=27 \pi \Gamma\left(\Upsilon \rightarrow e^{+} e^{-}\right) / 2 \alpha^{2}$ with $\Gamma\left(\Upsilon \rightarrow e^{+} e^{-}\right)=1.32 \mathrm{keV}$ while $\Gamma=52.5 \mathrm{keV}$ is its full width. The singular contribution of the $\Upsilon$-resonance to the polarization function abruptly changes between the two extremes taken from eq. (22)

$$
\pm \frac{2}{3} m_{\Upsilon} \Delta_{\Upsilon}\left(\frac{1}{2 \Gamma m_{\Upsilon}}\right)
$$

as the normalization point passes the position of the resonance. Numerically one gets

$$
\frac{\Delta_{\Upsilon}}{3 \Gamma}=6.7 \times 10^{3}
$$

which is far too big from the point of view of phenomenology.

The examples presented in this paper clearly demonstrate the inconsistency of the present definition of the running electromagnetic coupling constant within the principal value prescription. These problems are not noticeable, however, when one discusses a normalization point around $M_{Z}$ because the hadronic spectral density is smooth in this region.

We are going to present the results of an accurate numerical analysis within the Euclidean definition elsewhere. 
To conclude, we suggest that the Euclidean domain definition of the running electromagnetic coupling constant at $M_{Z}$ is used as a reference parameter for high precision tests of the standard model at the $Z$ boson peak. It is free of the shortcomings of the present definition which is based on the propagator at the physical value of the $Z$ boson mass within the principal value prescription.

\section{Acknowledgements}

The work is supported in part by Volkswagen Foundation under contract No. I/73611. A.A. Pivovarov is supported in part by the Russian Fund for Basic Research under contracts Nos. 96-01-01860 and 97-02-17065. The present stay of A.A. Pivovarov in Mainz was made possible due to Alexander von Humboldt fellowship.

\section{References}

[1] Precision tests of the standard electroweak model. Ed. P. Langacker, World Scientific, 1995.

[2] D. Bardin et al., Electroweak working group report. CERN-95-03A, Sep 1997. $158 \mathrm{pp}$.

[3] W. Hollik, J. Phys. G23 (1997) 1503

[4] Particle Data Group, Eur. Phys. J. C3 (1998) 1

[5] E.C.G. Stueckelberg, A. Petermann, Helv. Phys. Acta 26 (1953) 499

[6] M. Gell-Mann, F.E. Low, Phys. Rev. 95 (1954) 1300

[7] J.Gunion et al., Frontiers in Physics, Addison-Wesley, Menlo Park, 1990

[8] M. Davier, LAL-98-87, Dec 1998. 13pp. hep-ph/9812370 
[9] S. Groote, J.G. Körner, K. Schilcher, and N.F. Nasrallah, Phys. Lett. B440 (1998) 375

[10] J.H. Kuhn, M. Steinhauser, Phys. Lett. B437 (1998) 425

[11] M. Davier, A. Hocker, Phys. Lett. B435 (1998) 427

[12] N.V. Krasnikov, R. Rodenberg, Nuovo Cim. 111A (1998) 217

[13] V. Novikov, L. Okun, M. Vysotsky, A. Rozanov, ITEP-PH-6-97, Jul 1997. 13p, hep-ph/9707359

[14] H. Burkhardt and B. Pietrzyk, Phys. Lett. B356 (1995) 398

[15] S. Eidelman and F. Jegerlehner, Z. Phys. C67 (1995) 585

[16] P. Langacker, Phys. Rept. 72 (1981) 185

[17] H.P. Nilles, Phys. Rept. 110 (1984) 1

[18] A. Bottino and C.W. Kim, Lett. Nuovo Cim. 30 (1981) 353; Nuovo Cim. 57A (1980) 459;

N.V. Krasnikov and A.A. Pivovarov, Phys. Lett. 116 B (1982) 168;

M.R. Pennington and G.G. Ross, Phys. Lett. 102 B (1981) 167;

M.R. Pennington, R.G. Roberts and G.G. Ross, Nucl. Phys. B242 (1984) 69

[19] A.A. Pivovarov, Sov. J. Nucl. Phys. 54 (1991) 676;

Z. Phys. C53 (1992) 461; Nuovo Cim. 105A (1992) 813

[20] S. Groote, J.G. Korner, A.A. Pivovarov, Phys. Lett. B407 (1997) 66; Mod. Phys. Lett. A13 (1998) 637

[21] K.G. Chetyrkin, J.H. Kühn and A. Kwiatkowski, Phys. Rep. 277 (1996) 189 
[22] M.A. Shifman, A.I. Vainshtein and V.I. Zakharov, Nucl. Phys. B147 (1979) 385

[23] N.F. Nasrallah, N.A.Papadopoulos and K. Schilcher, Z. Phys. C16 (1983) 323

[24] K.G. Chetyrkin, A. Kwiatkowski, Z. Phys. C59 (1993) 525

[25] See W.Hollik contribution in [1], p.37.

[26] N.V. Krasnikov, A.A. Pivovarov, N. Tavkhelidze, Z. Phys. C19 (1983) 301

[27] N.V. Krasnikov, A.A. Pivovarov, Phys. Lett. 112B (1982) 397 